\title{
A new derivation of Lüscher F-term and fluctuations around the giant magnon
}

\author{
Michał P. Heller, Romuald A. Janik ${ }^{\dagger}$ \\ and Tomasz Eukowski ${ }^{\ddagger}$ \\ Institute of Physics \\ Jagellonian University, \\ ul. Reymonta 4, \\ 30-059 Kraków \\ Poland \\ June 28, 2021
}

\begin{abstract}
In this paper we give a new derivation of the generalized Lüscher F-term formula from a summation over quadratic fluctuations around a given soliton. The result is very general providing that S-matrix is diagonal and is valid for arbitrary dispersion relation. We then apply this formalism to compute the leading finite size corrections to the giant magnon dispersion relation coming from quantum fluctuations.
\end{abstract}

\section{Introduction}

The discovery of integrability in the context of the AdS/CFT correspondence [1] which appeared both on the gauge theory side [2, 3, 4, 5, 6] and on the

*e-mail: heller@th.if.uj.edu.pl

$\dagger$ e-mail: ufrjanik@if.uj.edu.pl

$\ddagger$ e-mail: tomaszlukowski@gmail.com 
string theory side [7] gives a hope for finding, in principle, the exact spectrum of the quantized superstring in $A d S_{5} \times S^{5}$ and equivalently the spectrum of anomalous dimensions of all operators in $\mathcal{N}=4$ Super Yang-Mills theory.

A lot of progress has been done in the setting of infinitely long strings (strings with large charges/angular momenta) or very long gauge theory operators. The S-matrix for elementary excitations has been identified, initially in various subsectors [8, 9] and then for the full multiplet of elementary excitations [10]. The remaining overall scalar function - the so-called dressing factor [11, 12] - has been finally fixed in [13, 14 satisfying constraints of crossing symmetry [15].

Despite the immense progress a lot remains to be understood concerning the structure of energy levels of strings with finite charges or short operators. On the gauge theory side the problem was identified with wrapping interactions [16]. On the string side of the duality, these correspond to virtual corrections coming from particles propagating around the string worldsheet cylinder [17]. These corrections lead to effects which go beyond the asymptotic Bethe ansatz. Such phenomena have been observed in various calculations [18, 19, 20, 21, 22] and models [23, 24]. Currently intensive work is being done both at weak [25, 26, 27] and at strong coupling [28]. At strong coupling the finite size effects come (roughly) in two varieties

$$
\delta \varepsilon \propto e^{-\frac{2 \pi J}{\sqrt{\lambda} \sin \frac{p}{2}}}
$$

as for the classical finite size correction to the giant magnon [20], and

$$
\delta \varepsilon \propto e^{-\frac{2 \pi J}{\sqrt{\lambda}}}
$$

which typically arises from a summation over quadratic fluctuations around a classical string solution.

In [29], generalizations of Lüscher formulas [30] were derived for finding the leading finite-size correction to the dispersion relation of elementary excitations. There are two types of contributions: the $\mu$-term and the $F$-term. The first corresponds to a particle splitting into two on-shell particles which cross the cylinder and recombine. It is intimately related to the existence of bound states. At strong coupling it gives the correction

$$
\delta \varepsilon^{\mu}=-\frac{\sqrt{\lambda}}{\pi} \cdot \frac{4}{e^{2}} \cdot \sin ^{3} \frac{p}{2} \cdot e^{-\frac{2 \pi J}{\sqrt{\lambda} \sin \frac{p}{2}}}
$$


which exactly reproduces the classical computation of the leading finite-size giant magnon dispersion relation of [20]. Other checks have been done dealing with dyonic magnons [31.

The motivation for this work was to explore the role of the $F$-term for the giant magnon. One expects that summing the energies of small fluctuations around the giant magnon solution should give a correction of the type (2) which should be reproduced by the $F$-term formula 1

$$
\delta \varepsilon_{a}^{F}=-\int_{-\infty}^{\infty} \frac{d q}{2 \pi}\left(1-\frac{\varepsilon^{\prime}(p)}{\varepsilon^{\prime}\left(q_{*}\right)}\right) \cdot e^{-i q_{*} L} \cdot \sum_{b}(-1)^{F_{b}}\left(S_{b a}^{b a}\left(q_{*}, p\right)-1\right)
$$

Here $q$ is the original euclidean energy which plays the role of momentum in the space-time interchanged theory, $E=\varepsilon(p)$ is the dispersion relation and $q_{*}$ is determined by the Euclidean on-shell condition

$$
q^{2}+\varepsilon^{2}\left(q_{*}\right)=0
$$

It will be convenient to change integration variables and rewrite (44) as

$$
\delta \varepsilon_{a}^{F}=\int_{-\infty}^{\infty} \frac{\mathrm{d} q_{*}}{2 \pi i}\left(\varepsilon^{\prime}\left(q_{*}\right)-\varepsilon^{\prime}(p)\right) \cdot e^{-i q_{*} L} \cdot \sum_{b}(-1)^{F_{b}}\left(S_{b a}^{b a}\left(q_{*}, p\right)-1\right)
$$

where we used the relation

$$
q= \pm i \varepsilon\left(q_{*}\right)
$$

and we choose the plus sign.

In the course of performing the calculations we found a very close link of the generalized formula (4) or (6) with the summation over energies of small fluctuations. Indeed we found that the whole expression (6) can be exactly reproduced from a summation over quadratic fluctuations provided one uses exact scattering phase-shifts (and not just semiclassical ones). The result is very general and does not depend on the form of the dispersion relation but is true only in the case of diagonal scattering.

\footnotetext{
${ }^{1}$ The $(-1)^{F_{b}}$ missing in the original derivation of 29 has been independently observed in 36. Diagrammatically the $(-1)^{F_{b}}$ arises from a -1 factor due to a fermion loop in the 1PI self-energy. It can be compensated by another -1 if the fermions are antiperiodic on the cylinder (in the TBA interpretation this corresponds to a computation of the thermal partition function) but this does not happen here and the TBA interpretation is rather the computation of an index.
} 
The plan of this paper is as follows. In section 2 we will present the setup of the quadratic fluctuation calculation. In section 3 we will perform a Poisson resummation over the energies and recover (6). In section 4 we will apply the above formalism to find the leading quantum correction to the giant magnon. We will close the paper with a discussion.

\section{Quadratic fluctuations}

The giant magnon solution of [32], when presented in an appropriate gauge looks like a localized soliton. The spatial extent of the solution is $J$ (or $J+a E$ depending on the choice of light-cone gauge) and the original solution of 32 was defined on the infinite line i.e. with $J=\infty$. Once we consider finite but large $J$, the solution will be deformed and the resulting correction to the energy was found in [20]. If we want to compute the sum of energies of quadratic fluctuations, in principle we should consider fluctuations around the finite $J$ solution.

Let us note, however, that according to Lüscher formulas the leading finite size corrections appear with definite exponential terms which differ between the $\mu$-term, associated more with deformations of a classical solution and the $F$-term which appears to have an exponential term characteristic of quadratic fluctuations around the spinning string. As in this paper we want to concentrate on the term with the latter characteristics, we will neglect the impact of the deformation of the solution at finite $J$ on the energies of fluctuation modes. Such an effect would have generically an exponential factor of the $\mu$-term type.

We will hence consider fluctuations around the infinite $J$ magnon and put them on a cylinder with periodic boundary conditions of circumference $J$. For general theories there is also a clear distinction between $\mu$ and $F$-term exponential scalings so we will proceed with this assumption.

The setup is in fact very similar to the one considered in the paper 33] where effects of fluctuations around the $J=\infty$ magnon were analyzed. However putting the fluctuations on a cylinder in [33] was only a regularization procedure prior to the limit $J \rightarrow \infty$. Here we would like to argue that it can also be used to obtain leading finite-size effects of quantum fluctuations.

Since the soliton is localized, very far from the soliton core the fluctuation will be just like a fluctutation around the vacuum, hence in this case

another soliton. Now we have to impose the periodicity condition for the 
wave function of the 'fluctuation soliton'. As it will pass around the cylinder it will get an additional phase shift from scattering with the 'giant magnon' which can be directly expressed in terms of the forward S-matrix:

$$
S_{b a}^{b a}(k, p)=e^{i \delta_{b a}(k, p)}
$$

where the original soliton ('giant magnon') is of type 'a', while the fluctuation is an excitation of type ' $\mathrm{b}$ '. We assume that $e^{i \delta_{b a}(k, p)}$ is a pure phase, which is true for diagonal scattering and for small fluctuations around the giant magnon (see Eqns 115-117 in [33]). The quantization condition then reads

$$
k_{n}=\frac{2 \pi n}{L}+\frac{\delta_{b}\left(k_{n}\right)}{L}
$$

where we denote the circumference by $L$ and we suppress the explicit $p$ and $a$ dependence of $\delta_{b a}(k, p)$. The scattering phase is taken at $L=\infty$ because the corrections to $\delta_{b a}$ vanishing at infinity will be subleading to our result. A summation over the zero-point energies would then be

$$
\delta \varepsilon_{\text {naive }}=\frac{1}{2} \sum_{b} \sum_{n=-\infty}^{\infty}(-1)^{F_{b}}\left(\varepsilon\left(k_{n}\right)-\varepsilon\left(k_{n}^{(0)}\right)\right)
$$

where we subtracted off fluctuations around the vacuum with the standard momenta

$$
k_{n}^{0}=\frac{2 \pi n}{L}
$$

We will argue that to have agreement with the $F$-term computation we still have to slightly modify (10).

In the present context, the giant magnon is not stationary but is moving with velocity

$$
v=\frac{d \varepsilon(p)}{d p}
$$

The whole system is periodic if one considers together time translations $t \rightarrow$ $t+\tau$ and space translation $x \rightarrow x+L$, where the period is $\tau=L / v$. The analogs of the standard phase factor $\omega \tau \equiv \varepsilon(k) \tau$ are now the stability angles 34

$$
\nu(k)=\tau \varepsilon(k)+k L \equiv \tau \varepsilon(k)+\delta(k)=\tau\left(\varepsilon(k)+\frac{v}{L} \delta(k)\right)
$$


where we used the fact that $e^{2 \pi i n}=1$. This suggests that the correct quantity to sum is

$$
\delta \varepsilon_{\text {final }}=\frac{1}{2} \sum_{b} \sum_{n=-\infty}^{\infty}(-1)^{F_{b}}\left(\varepsilon\left(k_{n}\right)+\frac{v}{L} \delta\left(k_{n}\right)\right)-(\text { vacuum })
$$

We will proceed with this assumption and show that the generalized $F$-term formula (4) is exactly the leading exponential behaviour of the above sum. In the derivation we will just make a mild assumption that $\varepsilon(k)$ is a symmetric function of $k$.

\section{Summation over fluctuations}

The main technical obstacle in calculating the sum (14) is that in almost all cases we are unable to solve analytically the quantization conditions for the momenta (9). However this problem may be bypassed by writing an iterative but exact solution. Let us denote the combination $2 \pi n / L$ by $t$. Then it is clear that an exact solution of (9) is

$$
k(t)=t+\frac{\delta\left(t+\frac{\delta(t+\ldots)}{L}\right)}{L}
$$

Now we can perform a Poisson resummation of the sum over $n$ :

$$
\sum_{n=-\infty}^{\infty} F\left(\frac{2 \pi n}{L}\right)=\frac{L}{2 \pi} \sum_{m=-\infty}^{\infty} \int_{-\infty}^{+\infty} F(t) e^{-i m L t} d t
$$

\section{Lüscher formulas from fluctuations}

In this subsection we will concentrate on the terms which give the leading exponential large $L$ corrections, namely terms with $m= \pm 1$. As a result we will get the Lüscher's F-term. It turns out that it is possible to include also subleading terms in a closed form (see the next subsection).

Let us first consider the summation over energies in (14). To save space we will reinstate the summation over types of fluctuations and $(-1)^{F_{b}}$ at the end of the calculation. We thus have

$$
\delta \varepsilon_{1}=\frac{L}{4 \pi} \int_{-\infty}^{+\infty} e^{i L t}(\epsilon(k(t))-\epsilon(t)) d t+\frac{L}{4 \pi} \int_{-\infty}^{+\infty} e^{-i L t}(\epsilon(k(t))-\epsilon(t)) d t
$$


Now after an integration by parts, we obtain

$$
-\frac{1}{4 \pi i} \int_{-\infty}^{+\infty} e^{i L t} \epsilon^{\prime}(k(t)) \frac{d k}{d t} d t+\frac{1}{4 \pi i} \int_{-\infty}^{+\infty} e^{-i L t} \epsilon^{\prime}(k(t)) \frac{d k}{d t} d t-\frac{1}{2 \pi i} \int_{-\infty}^{+\infty} e^{-i L t} \epsilon^{\prime}(t) d t
$$

The next and key step is to change the integration variables in the first two integrals from $t$ to $k$ and use the functional equation $k(t)=t+\delta(k(t)) / L$. The result is

$$
-\frac{1}{4 \pi i} \int_{-\infty}^{+\infty} e^{i L\left(k-\frac{\delta(k)}{L}\right)} \epsilon^{\prime}(k) d k+\frac{1}{4 \pi i} \int_{-\infty}^{+\infty} e^{-i L\left(k-\frac{\delta(k)}{L}\right)} \epsilon^{\prime}(k) d k-\frac{1}{2 \pi i} \int_{-\infty}^{+\infty} e^{i L t} \epsilon^{\prime}(t) d t
$$

Since $\epsilon^{\prime}(t)$ is antisymmetric this can be rewritten as

$$
\delta \varepsilon_{1}=\frac{1}{4 \pi i} \int_{-\infty}^{+\infty} e^{-i L k}\left(e^{i \delta(k)}+e^{-i \delta(-k)}-2\right) \epsilon^{\prime}(k) d k
$$

Using the relation between the forward $S$ matrix and the phase shifts we obtain something very similar to the $F$-term:

$$
\delta \varepsilon_{1}=\frac{1}{4 \pi i} \int_{-\infty}^{+\infty} e^{-i L k}\left(S_{b a}^{b a}(k, p)+\left(S^{-1}\right)_{b a}^{b a}(-k, p)-2\right) \epsilon^{\prime}(k) d k
$$

where we use the relation

$$
\left(S^{-1}\right)_{a b}^{a b}(k, p)=\frac{1}{S_{a b}^{a b}(k, p)}
$$

which is valid for diagonal matrices. We will now show that the second term in the above equation, when summed over all flavours with $(-1)^{F_{b}}$ is in fact equal to the first one.

$$
\sum_{b}(-1)^{F_{b}} \cdot\left(S^{-1}\right)_{b a}^{b a}(-k, p)=\sum_{b}(-1)^{F_{b}} S_{b a}^{b a}(k, p)
$$

This equality comes from using crossing symmetry of the S-matrix

$$
\left(C^{-1} \otimes 1\right) S^{s t_{1}}\left(p_{1}, p_{2}\right)(C \otimes 1) S\left(-p_{1}, p_{2}\right)=1
$$

where $C$ is charge conjugation matrix and ${ }^{s t_{1}}$ denotes the supertranspose in the first entry of $\mathrm{S}$ :

$$
\left(S^{s t_{1}}\right)_{a_{1} a_{2}}^{b_{1} b_{2}}=(-1)^{F_{a_{1}} F_{b_{1}}+F_{a_{1}}} S_{b_{1} a_{2}}^{a_{1} b_{2}}
$$


Using crossing symmetry (24) we can rewrite

$$
\sum_{b}(-1)^{F_{b}} \cdot\left(S^{-1}\right)_{b a}^{b a}(-k, p)=s \operatorname{Tr}_{1}\left(C^{-1} S^{s t_{1}}(k, p) C\right)_{a}^{a}
$$

where $s T r_{1}$ denotes the supertrace with respect to the first entry of S. Using the properties of supertrace we can change the order of the matrices to obtain

$$
s \operatorname{Tr}_{1}\left(C^{-1} S^{s t_{1}}(k, p) C\right)_{a}^{a}=s \operatorname{Tr}_{1}\left(C C^{-1} S^{s t_{1}}(k, p)\right)_{a}^{a}=\sum_{b}(-1)^{F_{b}} S_{b a}^{b a}(k, p)
$$

where in the last equality we used the fact that $(-1)^{F_{b}} F_{b}+F_{b}=1$. Taking into account the above we obtain finally for $\delta \varepsilon_{1}$ :

$$
\delta \varepsilon_{1}=\frac{1}{2 \pi i} \int_{-\infty}^{+\infty} \epsilon^{\prime}(k) e^{-i L k} \sum_{b}(-1)^{F_{b}}\left(S_{b a}^{b a}(k, p)-1\right) d k
$$

In order to transform the above integral into a $F$-term like integral one has to shift the contour of integration in the same way as in the derivation of the $F$-term so as to make the momentum to be purely imaginary. The question of boundary terms is nontrivial and has to be considered on a case by case basis. However it certainly works for relativistic theories and, more importantly in the present context, it also works for a fermion system with the giant magnon dispersion relation (see [17]). Incidentally the integrality of $L$ was necessary there. It would be interesting to study this point further.

Note that at this stage we are missing the second piece of (6). We will show now that it comes from performing Poisson resummation of the second term in (14).

To this end we have to evaluate

$$
\delta \varepsilon_{2}=\frac{\epsilon^{\prime}(p)}{4 \pi} \int_{-\infty}^{+\infty} e^{i L t} \delta(k(t)) d t+\frac{\epsilon^{\prime}(p)}{4 \pi} \int_{-\infty}^{+\infty} e^{-i L t} \delta(k(t)) d t
$$

Using the quantization condition (91) it is convenient to express $\delta(k(t))$ as

$$
\delta(k(t))=k(t)-t
$$

Plugging it into the above integral and performing similar manipulations as for $\delta \varepsilon_{1}$, we arrive at

$$
\delta \varepsilon_{2}=\frac{\epsilon^{\prime}(p)}{4 \pi i} \int_{-\infty}^{+\infty} e^{-i L k}\left(e^{i \delta(k)}+e^{-i \delta(-k)}-2\right) d k
$$


Combining this contribution with (20), expressing the phase shifts through the $S$-matrix, rotating contours, using the equality (23) we arrive at the complete expression for the $F$-term:

$$
\delta \varepsilon_{1}+\delta \varepsilon_{2}=\int_{-\infty}^{\infty} \frac{\mathrm{d} q_{*}}{2 \pi i}\left(\varepsilon^{\prime}\left(q_{*}\right)-\varepsilon^{\prime}(p)\right) \cdot e^{-i q_{*} L} \cdot \sum_{b}(-1)^{F_{b}}\left(S_{b a}^{b a}\left(q_{*}, p\right)-1\right)
$$

The above derivation shows that Lüscher's $F$-term is equivalent to a summation over fluctuations. In order to recover the full expression we have to consider energies derived from stability angles. A special case of the above formula for a relativistic dispersion relation leads to the formula of [35] for corrections to a moving particle, while further specializing to a particle at rest reduces to the classical Lüscher formula [30]. In the following section we will apply this formalism to calculate the leading correction to the giant magnon dispersion relation coming from quadratic fluctuations.

\section{Refinements}

It is straightforward to compute contribution to the energy shift from the terms with arbitrary value of $m>02$ As a result we get

$$
\delta \varepsilon^{(m)}=\frac{1}{4 \pi i m} \int_{-\infty}^{+\infty} e^{-i m L k}\left(\epsilon^{\prime}(k)-\epsilon^{\prime}(p)\right)\left(e^{i m \delta(k)}+e^{-i m \delta(-k)}-2\right) d k
$$

where $m$ has the interpretation of the winding number associated with the virtual soliton going around the cylinder and interacting $m$-times with the giant magnon (which is still a 1-loop result). The Lüscher's F-term is reproduced by $m=1$ (this corresponds to a single interaction with a virtual particle).

In order to obtain the Poisson-resummed 1-loop energy shift we have to sum over all values of $m$. After the identification (8) and using crossing symmetry the final formula reads

$$
\sum_{m=1}^{+\infty} \delta \varepsilon^{(m)}=-\int_{-\infty}^{\infty} \frac{\mathrm{d} q_{*}}{2 \pi i}\left(\varepsilon^{\prime}\left(q_{*}\right)-\varepsilon^{\prime}(p)\right) \sum_{b}(-1)^{F_{b}} \log \left(\frac{1-S_{b a}^{b a}\left(q_{*}, p\right) e^{-i q_{*} L}}{1-e^{-i q_{*} L}}\right)
$$

\footnotetext{
${ }^{2}$ The case $m=0$ is trivial. There is no contribution to the energy shift under the assumption that $\sum_{b}(-1)^{F_{b}} \delta_{b a}(k)=0$ for any $a$ and $k$ (this is true for the giant magnon phase shifts).
} 
We have to keep in mind that terms with higher $m$ will appear at the same order as contributions from higher loop processes which are not taken into account by the sum over fluctuation energies.

Let us now return to the formulas (28) and (31). These provide integral formulas equivalent to Lüscher formulas but defined as integrals over physical real momenta. Although our derivation and interpretation in terms of fluctuations fails for general S-matrices with nondiagonal scattering we found that one can give a similar integral formula which is valid also in these other cases (a typical example would be e.g. the $\mathrm{O}(3)$ model)

$$
\delta \varepsilon=\frac{1}{2 \pi i} \int d k\left(\varepsilon^{\prime}(k)-\varepsilon^{\prime}(p)\right) e^{-i k L} \sum_{b}(-1)^{F_{b}}\left(S_{b a}^{b a}(k, p)-1\right)
$$

\section{The giant magnon}

Let us now proceed to compute the leading finite size correction to the giant magnon dispersion relation coming from quantum fluctuations. We will perform a saddle point calculation of the $F$-term integral. The exponent in the $F$-term formula is

$$
e^{-2 J \operatorname{arcsinh}\left(\frac{1}{4 g} \sqrt{1+q^{2}}\right)}
$$

where we use the conventions of [14] i.e. $g=\sqrt{\lambda} /(4 \pi)$. Saddle point expansion gives

$$
e^{-\frac{J}{2 g}-\frac{J}{4 g} q^{2}}
$$

The saddle point has Euclidean energy $q=0$. Gaussian integration gives

$$
2 \sqrt{\frac{\pi g}{J}} e^{-\frac{J}{2 g}}=e^{-\frac{2 \pi J}{\sqrt{\lambda}}} \cdot \frac{\lambda^{\frac{1}{4}}}{\sqrt{J}}
$$

The rest of the integrand has to be evaluated at the saddle point $q=0$. The Jacobian factor is then trivial

$$
\left(1-\frac{\varepsilon^{\prime}(p)}{\varepsilon^{\prime}\left(q_{*}\right)}\right) \rightarrow 1
$$

The phase shifts have to be evaluated for the virtual particle at the saddle point:

$$
x_{q}^{+}=i\left(1+\frac{1}{4 g}\right) \quad x_{q}^{-}=i\left(1-\frac{1}{4 g}\right)
$$


and for the giant magnon described by the strong coupling expressions:

$$
x_{p}^{+}=e^{\frac{i p}{2}}\left(1+\frac{1}{4 g \sin \frac{p}{2}}\right) \quad x_{p}^{-}=e^{\frac{-i p}{2}}\left(1+\frac{1}{4 g \sin \frac{p}{2}}\right)
$$

Now we may evaluate the phase shifts using (8). The contribution of the dressing phase at the saddle point reduces, in the strong coupling limit, to the contribution of the AFS phase [11] which gives

$$
\frac{1+\sin \frac{p}{2}}{1-\sin \frac{p}{2}} e^{-i p} e^{-2 \sin \frac{p}{2}}
$$

The phase shifts of the $S^{5}$ scalars, $A d S_{5}$ scalars and fermions (see Eqns $36-41$ in [33]) then evaluate to

$$
\begin{aligned}
\left(e^{i \delta}\right)_{S^{5}} & =\frac{1+\sin \frac{p}{2}}{1-\sin \frac{p}{2}} \cdot e^{-2 \sin \frac{p}{2}} \\
\left(e^{i \delta}\right)_{A d S_{5}} & =1 \cdot e^{-2 \sin \frac{p}{2}} \\
\left(e^{i \delta}\right)_{\text {fermions }} & =\frac{\cos \frac{p}{4}+\sin \frac{p}{4}}{\cos \frac{p}{4}-\sin \frac{p}{4}} \cdot e^{-2 \sin \frac{p}{2}}
\end{aligned}
$$

Summing the phase shifts $\left(4\left(e^{i \delta}\right)_{S^{5}}+4\left(e^{i \delta}\right)_{A d S_{5}}-8\left(e^{i \delta}\right)_{\text {fermions }}\right)$ gives the final expression

$$
\delta \varepsilon^{F}=-\frac{1}{2 \pi} \cdot \frac{\lambda^{\frac{1}{4}}}{\sqrt{J}} \cdot \frac{16 \sin ^{2} \frac{p}{4}}{1-\sin \frac{p}{2}} e^{-2 \sin \frac{p}{2}} e^{-\frac{2 \pi J}{\sqrt{\lambda}}}
$$

The above procedure is of course equivalent to performing a saddle point directly in the Poisson resummed expression for a sum over fluctuation energies. To this order of approximation it is enough to consider relativistic dispersion relation for the fluctuations as in [33], $\varepsilon(k)=\sqrt{1+k^{2}}$. Performing a change of variables to $k=\frac{2 r}{1-r^{2}}$ one can evaluate the saddle point to $r=i$. The derivation in section 3 however, identifies directly the sum over fluctuations which corresponds to the $F$-term formula in its full generality without the need for a saddle point approximation.

\section{Discussion}

In this paper we have shown that the generalized Lüscher $F$-term formula (44) has a very transparent interpretation as the leading exponential term in 
a summation over frequencies derived from stability angles. For a particle at rest, corresponding to the standard Lüscher calculation, the summation is just over zero-point energies. For a moving particle, the modifications due to the stability angles are necessary. Hence under very mild assumptions such calculations of 1-loop effects have to agree.

We have used the above formalism to evaluate the leading finite size correction to the giant magnon dispersion relation coming from quantum fluctuations. By the above reasoning the F-term computation and the summation over modes are by definition really identical computations.

It would be interesting to understand more deeply the appearance of these effective energies derived from stability angles in this context especially as the relation to WKB methods is not entirely clear here. We leave this problem for future research.

Acknowledgments. RJ would like to thank Zoltan Bajnok and Sakura Schäfer-Nameki for interesting discussions. This work has been supported in part by Polish Ministry of Science and Information Technologies grant 1P03B04029 (2005-2008), RTN network ENRAGE MRTN-CT-2004-005616, and the Marie Curie ToK KraGeoMP (SPB 189/6.PR UE/2007/7).

Note added: As this paper was being completed, an interesting paper [36] appeared were quantum fluctuations around the giant magnon were calculated and compared with the $F$-term using different methods.

\section{References}

[1] J. M. Maldacena, "The large N limit of superconformal field theories and supergravity," Adv. Theor. Math. Phys. 2 (1998) 231 [Int. J. Theor. Phys. 38 (1999) 1113], hep-th/9711200];

S. S. Gubser, I. R. Klebanov and A. M. Polyakov, "Gauge theory correlators from non-critical string theory," Phys. Lett. B 428 (1998) 105, hep-th/9802109;

E. Witten, "Anti-de Sitter space and holography," Adv. Theor. Math. Phys. 2 (1998) 253, hep-th/9802150.

[2] J. A. Minahan and K. Zarembo, "The Bethe-ansatz for $\mathrm{N}=4$ super Yang-Mills," JHEP 0303, 013 (2003), hep-th/0212208. 
[3] N. Beisert, C. Kristjansen and M. Staudacher, "The dilatation operator of $\mathrm{N}=4$ super Yang-Mills theory," Nucl. Phys. B 664 (2003) 131, hep-th/0303060.

[4] N. Beisert and M. Staudacher, "The N = 4 SYM integrable super spin chain," Nucl. Phys. B 670 (2003) 439, hep-th/0307042.

[5] A. V. Belitsky, S. E. Derkachov, G. P. Korchemsky and A. N. Manashov, "Quantum integrability in (super) Yang-Mills theory on the light-cone," Phys. Lett. B 594 (2004) 385 [hep-th/0403085].

[6] L. Dolan, C. R. Nappi and E. Witten, "A relation between approaches to integrability in superconformal Yang-Mills theory," JHEP 0310 (2003) 017, hep-th/0308089.

[7] I. Bena, J. Polchinski and R. Roiban, "Hidden symmetries of the AdS(5) x S**5 superstring," Phys. Rev. D 69 (2004) 046002, hep-th/0305116.

[8] M. Staudacher, "The factorized S-matrix of CFT/AdS," JHEP 0505, 054 (2005) hep-th/0412188.

[9] N. Beisert and M. Staudacher, "Long-range PSU $(2,2 \mid 4)$ Bethe ansaetze for gauge theory and strings," Nucl. Phys. B 727, 1 (2005) hep-th/0504190.

[10] N. Beisert, "The su(2-2) dynamic S-matrix," arXiv:hep-th/0511082.

[11] G. Arutyunov, S. Frolov and M. Staudacher, "Bethe ansatz for quantum strings," JHEP 0410 (2004) 016 arXiv:hep-th/0406256.

[12] R. Hernandez and E. Lopez, "Quantum corrections to the string Bethe ansatz," JHEP 0607, 004 (2006) arXiv:hep-th/0603204.

[13] N. Beisert, R. Hernandez and E. Lopez, "A crossing-symmetric phase for AdS(5) x S**5 strings," JHEP 0611, 070 (2006) arXiv:hep-th/0609044].

[14] N. Beisert, B. Eden and M. Staudacher, "Transcendentality and crossing," J. Stat. Mech. 0701, P021 (2007) arXiv:hep-th/0610251.

[15] R. A. Janik, "The $\operatorname{AdS}(5)$ x $\mathrm{S}^{* *} 5$ superstring worldsheet Smatrix and crossing symmetry," Phys. Rev. D 73, 086006 (2006) arXiv:hep-th/0603038. 
[16] N. Beisert, V. Dippel and M. Staudacher, "A novel long range spin chain and planar $\mathrm{N}=4$ super Yang-Mills," JHEP 0407, 075 (2004) hep-th/0405001.

[17] J. Ambjorn, R. A. Janik and C. Kristjansen, "Wrapping interactions and a new source of corrections to the spin-chain / string duality," Nucl. Phys. B 736 (2006) 288 hep-th/0510171.

[18] S. Schafer-Nameki, "Exact expressions for quantum corrections to spinning strings," Phys. Lett. B 639 (2006) 571 arXiv:hep-th/0602214|.

[19] S. Schafer-Nameki, M. Zamaklar and K. Zarembo, "How accurate is the quantum string Bethe ansatz?," JHEP 0612, 020 (2006) arXiv:hep-th/0610250].

[20] G. Arutyunov, S. Frolov and M. Zamaklar, "Finite-size effects from giant magnons," Nucl. Phys. B 778, 1 (2007) arXiv:hep-th/0606126.

[21] D. Astolfi, V. Forini, G. Grignani and G. W. Semenoff, "Gauge invariant finite size spectrum of the giant magnon," Phys. Lett. B 651 (2007) 329 arXiv:hep-th/0702043.

[22] A. V. Kotikov, L. N. Lipatov, A. Rej, M. Staudacher and V. N. Velizhanin, "Dressing and Wrapping," arXiv:0704.3586 [hep-th].

[23] A. Rej, D. Serban and M. Staudacher, "Planar $N=4$ gauge theory and the Hubbard model," JHEP 0603, 018 (2006) arXiv:hep-th/0512077.

[24] G. Feverati, D. Fioravanti, P. Grinza and M. Rossi, "Hubbard's adventures in $\mathrm{N}=4$ SYM-land? Some non-perturbative considerations on finite length operators," J. Stat. Mech. 0702 (2007) P001 arXiv:hep-th/0611186.

[25] F. Fiamberti, A. Santambrogio, C. Sieg and D. Zanon, "Wrapping at four loops in N=4 SYM," arXiv:0712.3522 [hep-th].

[26] B. Eden, "Boxing with Konishi," arXiv:0712.3513 [hep-th].

[27] C. A. Keeler and N. Mann, "Wrapping Interactions and the Konishi Operator," arXiv:0801.1661 [hep-th]. 
[28] J. A. Minahan and O. O. Sax, "Finite size effects for giant magnons on physical strings," arXiv:0801.2064 [hep-th].

[29] R. A. Janik and T. Lukowski, "Wrapping interactions at strong coupling - the giant magnon," Phys. Rev. D 76, 126008 (2007) arXiv:0708.2208 [hep-th]].

[30] M. Lüscher, "Volume Dependence Of The Energy Spectrum In Massive Quantum Field Theories. 1. Stable Particle States," Commun. Math. Phys. 104 (1986) 177.

[31] Y. Hatsuda and R. Suzuki, "Finite-Size Effects for Dyonic Giant Magnons," arXiv:0801.0747 [hep-th].

[32] D. M. Hofman and J. M. Maldacena, "Giant magnons," J. Phys. A 39, 13095 (2006) arXiv:hep-th/0604135.

[33] H. Y. Chen, N. Dorey and R. F. Lima Matos, "Quantum Scattering of Giant Magnons," JHEP 0709, 106 (2007) [arXiv:0707.0668 [hep-th]].

[34] R. F. Dashen, B. Hasslacher and A. Neveu, "The Particle Spectrum In Model Field Theories From Semiclassical Functional Integral Techniques," Phys. Rev. D 11, 3424 (1975).

[35] T. R. Klassen and E. Melzer, "On The Relation Between Scattering Amplitudes And Finite Size Mass Corrections In Qft," Nucl. Phys. B 362 (1991) 329.

[36] N. Gromov, S. Schafer-Nameki and P. Vieira, "Quantum Wrapped Giant Magnon," arXiv:0801.3671 [hep-th]. 